\title{
TIDAL FRICTION IN EARLY-TYPE STARS
}

\author{
PeTER Goldreich \\ California Institute of Technology \\ AND \\ PHILIP D. NichOLSON \\ Cornell University \\ Received 1988 September 8; accepted 1988 December 29
}

\begin{abstract}
The tidal torque on an early-type star is concentrated near the boundary between the convective core and radiative envelope and a train of gravity waves is excited there. The angular momentum which the torque removes from the fluid is transported outward by the gravity waves, which carry negative angular momentum. Before the surface layers are despun to synchronous rotation, the gravity waves propagate to just below the photosphere where they suffer radiative damping and are partially reflected. It is here that the negative angular momentum is deposited and the primary tidal despinning takes place. The surface layers cannot be spun down below synchronous rotation because as a train of gravity waves approaches a corotation resonance its group velocity and wavelength tend to zero, its amplitude diverges, and it is completely absorbed. Thus, tidal despinning to synchronous rotation proceeds from the outside toward the inside of the star. Our picture provides a neat explanation for the otherwise puzzling discovery by Giuricin, Mardirossian, and Mezzetti that Zahn's theory for tidal evolution in early-type close binaries seems to be compatible with the observed rates of orbit circularization while significantly underestimating the observed rates of spin synchronization.
\end{abstract}

Subject headings: stars: binaries — stars: early-type — stars: rotation — wave motions

\section{INTRODUCTION}

The plan of this paper is as follows. In $\S$ II we present a brief description of the best theory of tides in early-type stars, that of Zahn $(1975,1977)$. Next, we summarize the current status of the comparison between the theory and the observations. This leads to the identification of a major difficulty, namely, the theory seems to provide reasonable time scales for circularization but to severely overestimate the time scales for synchronization, or pseudosynchronization. We apply a theorem proved in a companion paper (Goldreich and Nicholson 1989; hereafter Paper I) to resolve this puzzle by reinterpreting Zahn's theory. Because the theorem of Paper I is somewhat abstract, and also because all published treatments of tidal perturbations in early-type stars are rather imposing, we present a heuristic description of these perturbations in $\S$ III. This description provides a more concrete basis for our reinterpretation of Zahn's work. We also use it to clarify some points raised by Savonije and Papaloizou (1983) in their reexamination of Zahn's theory. Finally, in $\S$ IV we discuss some thorny issues related to differential rotation in stars that are being tidally despun and outline prospects for their resolution by future investigations.

\section{ZAHN'S THEORY}

We owe to Zahn $(1966 a, b, c, 1975,1977)$ much of our understanding of the mechanisms of tidal friction in stars. In particular, he showed that tidal forcing of an early-type star excites gravity waves at the boundary between the convective core and radiative envelope. Zahn argued convincingly that the torque associated with the excitation of gravity waves is much greater than that associated with turbulent viscosity in the convective core. The latter torque is negligible because the time scales of the dominant convective motions in the core are very long in comparison to the periods of tidal forcing. The situation is quite different in late-type stars. These stars have convection zones in their outer envelopes and the convective time scales are short enough that turbulent viscosity is likely to be the dominant process of tidal dissipation. One may quibble about the details, but the general picture of tidal dissipation presented by Zahn (1977) seems likely to be correct. In this paper we are only concerned with tidal friction in early-type stars, and so we mostly ignore the effects of turbulent viscosity.

Gravity waves are evanescent in the convectively unstable core and propagate in the stably stratified radiative envelope. The boundary between the core and envelope is a turning point where the WKBJ wavelength of gravity waves is formally infinite. Throughout the rest of the envelope the radial wavelength of tidally excited gravity waves is very short compared to the stellar radius. Thus, the tidal potential, which varies radially on the scale of the stellar radius, couples best to the gravity waves near the core envelope boundary. Indeed, virtually the entire net tidal torque is exerted in the immediate vicinity of this boundary. In $\S$ III we show that the angularly averaged tidal torque density is an oscillatory function of radius, with the net torque concentrated in a thin region, no more than a few wavelengths in width, near the outer boundary of the convective core.

In a uniformly rotating star the tidally excited gravity waves propagate out through the stellar envelope to the atmosphere where they suffer radiative damping and are thus only partially reflected. Zahn's calculations relate the radiative damping of the waves to the tidal torque. He then goes on to derive approximate formulae for the rates at which early-type stars in binaries despin and their orbits circularize. 


\section{a) Current Status}

Giuricin, Mardirossian, and Messetti $(1984 a, b, c)$ have collected and discussed observational evidence that bears on the rates of orbital circularization and spin synchronization in early-type binaries. They used published data and attempted to separate unevolved and evolved systems so that they could identify samples of binaries whose stars have not yet undergone mass exchange. Their study of circularization, based on a sample of $\sim 200$ eclipsing and double-lined binaries, leads them to conclude that Zahn's theory "appears to be substantially compatible" with the observed distributions of orbital eccentricity. Although binaries with large separations show a wide range of orbital eccentricity, including essentially circular orbits, there are virtually no eccentric orbits in systems with fractional radius (the ratio of primary stellar radius to orbital radius), $r>0.25$. Giuricin et al. (1984b) "emphasize that this finding appears to be substantially consistent with Zahn's theoretical predictions." They go on to conclude that near circular orbits with $r \ll 0.25$ reflect the initial distribution of eccentricities, which they suggest has a maximum at $e=0$. This picture is in accord with tidal evolution, which implies an absence of eccentric orbits in very close binaries but does not require that widely separated binaries necessarily have nonzero eccentricities. However, the authors conclude that Zahn's theory does not predict the high degree of synchronization, or pseudosynchronization, they find. In their words, it "appears to be clearly incompatible" with data from a sample of 140 eclipsing binaries and "inadequate to account" for the strong tendency for synchronization seen in a sample of 80 double-lined spectroscopic binaries.

The preceding paragraph summarizes the views of Giuricin et al. regarding the success of Zahn's tidal theory as applied to early-type binaries. However, it is clear that the existence of circular orbits for numerous binaries having $r \leq 0.20$ or $r \leq 0.15(40 \%$ or $31 \%)$ still requires an explanation. Giuricin $e t$ al. $(1984 b)$ advance the hypothesis that this reflects the initial distribution of orbital eccentricity, presumably at the time the stars first reach the main sequence. Of course, their hypothesis is just speculation. It is possible that a more powerful mechanism than that advocated by Zahn drives tidal evolution in a fraction of early-type binaries.

The mixed review that Zahn's theory receives when confronted by the observations is puzzling. Its success when applied to orbital circularization suggests that it is probably basically correct. Since observations of stellar spin rates all refer to photospheric layers, perhaps these spin down more rapidly than the stellar interiors. In the following subsection we argue that this is indeed likely.

\section{b) Reinterpretation of Zahn's Theory}

In the previous subsection we raised the possibility that while Zahn's theory of tidal evolution may be basically correct, it could be missing some critical ingredient. To investigate this possibility, we examine in more detail the manner in which tidal spin-down proceeds in early-type stars.

We have stated that the net tidal torque is concentrated near the boundary of the convective core, and that the gravity waves it excites propagate out through the radiative envelope toward the atmosphere. How then does the star despin? The answer to this question follows immediately from a theorem on tides in rotating fluids which we prove in Paper I. The theorem states that, in the absence of dissipation and away from corota- tion, tidal forcing does not produce any secular variation of the angular momenta of fluid elements. Both viscous and radiative damping of tidally excited gravity waves are expected to be negligible in the stellar interior. Moreover, corotation resonances are not involved in the initial stages of the despinning of those stars which begin life rotating at above the synchronous rate. ${ }^{1}$ Under these conditions our theorem implies that the gravity waves transport all of the negative angular momentum which the tidal torque deposits near the boundary of the convective core out to the stellar atmosphere. Only there, where the waves suffer radiative damping and are partially reflected, does the primary despinning occur.

\section{HEURISTIC DERIVATION OF TIDAL PERTURBATIONS}

We consider the tidal forcing of an early-type star of mass $M_{1}$ by a companion of mass $M_{2}$. To keep the exposition as simple as possible, we work to order of magnitude only and assume that $M_{1} \gg M_{2}$. We denote by $\Omega$ the spin angular velocity of the primary star. The relative orbit is taken to be circular and equatorial. The orbital radius, $a$, and angular velocity, $\omega$, are related by

$$
\omega=\left(\frac{G M_{1}}{a^{3}}\right)^{1 / 3} .
$$

The fluid particles are tidally forced at the Doppler-shifted frequency

$$
\sigma=2(\Omega-\omega) \text {. }
$$

We neglect the effect of the Coriolis acceleration on the tidal dynamics. Strictly speaking, this limits the applicability of our considerations to nonrotating stars for which $\sigma=-2 \omega$. However, we maintain the distinction between $\sigma$ and $-2 \omega$, and even pretend that $\sigma>0 .^{2}$

We are primarily concerned with low frequency tides, those for which

$$
\epsilon \equiv \frac{\sigma^{2}}{G \bar{\rho}(R)} \ll 1,
$$

where $\bar{\rho}(R)$ is the mean density of the primary star. $^{3}$ In this limit the tidal forcing frequency is much lower than the fundamental oscillation frequency of the star, and the tidal perturbation decomposes naturally into an equilibrium and a dynamical component. The equilibrium tide, which is the entire tide in the limit $\sigma=0$, is a prolate spheroidal distortion whose symmetry axis points toward the companion. The dynamical tide is a train of gravity waves which is excited at the boundary of the convective core and propagates outward. For the moment we make the simplifying assumption that the gravity waves are totally absorbed near the stellar surface, so there is no reflected, inward propagating wave train.

\section{a) Tidal Disturbance}

We assume an adiabatic tidal disturbance, which is likely to be valid everywhere except very near the stellar surface. The

\footnotetext{
1 The role of corotation resonances in the later stages of tidal despinning is discussed in $\S$ IV.

${ }^{2}$ The dynamical effects of the Coriolis acceleration on the tidal forcing of early-type stars are described by Nicholson (1978).

${ }^{3}$ Henceforth, we denote mean density by $\bar{\rho}$ without specifying a radius $r$. Local densities are denoted by $\rho$.
} 
tidal perturbation of a nonrotating star is governed by the linearized, Eulerian, perturbation equations

$$
\begin{gathered}
\delta \rho+\nabla \cdot(\rho \xi)=0, \\
\rho \frac{\partial^{2} \xi}{\partial t^{2}}=-\nabla \delta p-\delta \rho g-\rho \nabla U,
\end{gathered}
$$

and

$$
\delta p+\xi_{r} \frac{\partial p}{\partial r}=\frac{\gamma p}{\rho}\left(\delta \rho+\xi_{r} \frac{\partial \rho}{\partial r}\right),
$$

which express the conservation of mass, momentum, and entropy. We denote by $\delta$ Eulerian perturbations of quantities with nonvanishing equilibrium values, and by $\xi$ the vector field giving the displacements of fluid elements from their unperturbed positions. The perturbed gravitational field of the primary star has been neglected in equation (5). Its principal effect could be included by multiplying the tidal potential, $U$, by a function of $r$ which is slightly larger than unity. However, this is too fine a point to worry about here. Last, we assume the dependence $P_{2}^{2}(\cos \theta) \exp (i 2 \phi-i \sigma t)$ for all perturbation variables.

\section{i) Equilibrium Tide}

The equilibrium tidal perturbation is obtained by setting $\sigma=0$ and solving in succession the angular and then the radial component of the momentum equation (5), the entropy equation (6), and finally, the continuity equation (4). For the leading quadrupole-type term in the tidal potential, we find:

$$
\begin{gathered}
\delta p^{\mathrm{eq}}=-\rho U, \\
\delta \rho^{\mathrm{eq}}=\frac{U}{g} \frac{\partial \rho}{\partial r}, \\
\xi_{r}^{\mathrm{eq}}=-\frac{U}{g},
\end{gathered}
$$

and

$$
\xi_{\perp}^{\mathrm{eq}}=-\frac{1}{6 r^{2}} \frac{\partial}{\partial r}\left(\frac{r^{4}}{g}\right) \nabla_{\perp} U .
$$

The subscript $\perp$ denotes the projection of a vector, or vector operator, in the plane orthogonal to $r$.

\section{ii) Dynamical Tide}

The dynamical tide in the radiative envelope is a train of gravity waves. The properties of gravity waves are deduced by solving the perturbation equations with $U=0$. A great simplification results from making the approximation $r k_{r} \gg 1$, where $k_{r}$ is the radial wavevector of the gravity waves.

Eliminating $\xi_{r}$ from equations (4) and (5), and making use of equation (3), we obtain

$$
\delta \rho^{\mathrm{d}} \approx-i \frac{k_{r}}{g} \delta p^{\mathrm{d}}
$$

that is, the radial force balance is essentially hydrostatic. Substituting this relation into the equation of state yields

$$
\xi_{r}^{\mathrm{d}} \approx-i \frac{k_{r}}{\rho N^{2}} \delta p^{\mathrm{d}}
$$

where

$$
N^{2} \equiv \frac{g}{\gamma} \frac{\partial}{\partial r} \ln \left(\frac{p}{\rho \gamma}\right)
$$

is the square of the Brunt-Vaisala frequency. The angular component of equation (5) implies

$$
\xi_{\perp}^{\mathrm{d}}=\frac{1}{\sigma^{2} \rho} \nabla_{\perp} \delta p^{\mathrm{d}} .
$$

The dispersion relation follows from inserting equations (11)-(14) into the continuity equation (4) and using $\nabla_{\perp}^{2} \delta p^{d}=$ $-6 \delta p^{\mathrm{d}} / r^{2}$. It reads

$$
k_{\mathrm{r}}^{2} \approx \frac{6 N^{2}}{r^{2} \sigma^{2}}
$$

The displacement associated with the gravity wave is predominantly horizontal. From equations (12), (14), and (15), we find $O\left(\xi_{r}^{d} / \xi_{\perp}^{d}\right) \sim \sigma / N \ll 1$.

The radial component of the group velocity of the gravity waves,

$$
v_{g} \equiv \frac{\partial \sigma}{\partial k_{r}}=-\frac{\sigma}{k_{r}}
$$

is of equal magnitude but opposite sign to the radial component of the phase velocity. Note that the group velocity vanishes and the radial component of the wave vector diverges at a corotation resonance, i.e., as $\sigma \rightarrow 0$. Thus the waves can never reach the corotation resonance, but are damped by diffusive effects.

In massive main-sequence stars $N^{2}$ rises approximately linearly from 0 at the boundary of the convective core, $r=r_{c}$, until it approaches $\sim g / H$ for $r \gtrsim 1.5 r_{c}$. Here, $H$ is the local pressure scale height. ${ }^{4}$ This implies that the wavelength drops from

$$
\lambda_{1} \sim\left(\frac{\sigma^{2}}{G \bar{\rho}}\right)^{1 / 3} r
$$

just outside the core to

$$
\lambda(r) \sim\left(\frac{\sigma^{2}}{G \bar{\rho}}\right)^{1 / 2} r
$$

for $r \gtrsim 1.5 r_{c}$. A simple calculation shows that the time it takes to cross a wavelength while moving at the group speed is of order $\sim 1 / \sigma$, independent of position in the radiative envelope.

Next, we turn our attention to the manner in which the amplitudes of the perturbations associated with the gravity waves vary in the radiative envelope. We use a tilde ${ }^{\sim}$ to distinguish the amplitude of a perturbation variable, which is a function of $r$ only, from the complete perturbation variable, which is a function of $r, \theta, \phi$, and $t$.

Gravity waves carry a conserved luminosity of wave action. ${ }^{5}$ Both the energy and the angular momentum density of a wave are proportional to its action density. ${ }^{6}$

The energy luminosity, $L_{E}$, may be expressed as due to the radial transport of kinetic energy density at the group speed, ${ }^{7}$

$$
L_{E} \sim \rho r^{2} v_{g} \sigma^{2}\left(\tilde{\xi}_{\perp}^{\mathrm{d}}\right)^{2},
$$

or alternately as due to the energy flux associated with $p-v$ work.

$$
L_{E} \sim r^{2} \delta \tilde{p}^{\mathrm{d}} \tilde{v}_{r}^{\mathrm{d}}
$$

${ }^{4}$ We replace $H$ by $r$ except for near-surface layers.

${ }^{5}$ Luminosity is flux multiplied by $r^{2}$ and integrated over solid angle.

${ }^{6}$ The Jacobi relation implies that the ratio of the energy density to the angular momentum density is $\omega$, the pattern speed of the tidal potential.

${ }^{7}$ Recall that $\bar{\xi}_{\perp} \gg \bar{\xi}_{\text {r }}$. 
Using equations (11)-(14) it is easy to verify that the two expressions for $L_{E}$ are equivalent.

Introducing the dispersion relation given by equation (15) into the linearized perturbation equations (4)-(6), and eliminating all the dependent variables in favor of $\xi_{\perp}$, we obtain the WKBJ amplitude relation

$$
\frac{\rho r^{2}\left(\tilde{\xi}_{\perp}^{\mathrm{d}}\right)^{2}}{k_{r}}=\text { constant } .
$$

Equation (21) expresses the conservation of the luminosity of wave action. A comparison of equations (19) and (21) reveals that the former is just $-\sigma^{3}$ times the latter. We use equation (21) to obtain the radial variation of the gravity wave amplitude.

In Figure 1, a sample numerical solution for the dynamical tide in a nonrotating, $5 M_{\odot}$ main-sequence star is shown, after Nicholson (1978), together with the analytic, constantluminosity, WKBJ amplitude given by equation (21). Note that the full WKBJ amplitude is reached within about one wavelength from the core-envelope boundary.

\section{b) Tidal Torque}

To evaluate the angular momentum luminosity, $L_{H}=L_{E} / \omega$, carried by the train of tidally forced gravity waves, we set $\tilde{\xi}_{\perp}^{\mathrm{d}} \sim \tilde{\xi}_{\perp}^{\text {eq }}$ at a distance $\sim \lambda_{1}$ outside the core boundary. ${ }^{8}$ This yields

$$
L_{H} \sim-\bar{\rho} r^{5} \omega^{2} \frac{\omega \sigma^{11 / 3}}{(G \bar{\rho})^{7 / 3}}\left(\frac{M_{2}}{M_{1}}\right)^{2} .
$$

In writing equation (22) we have used Kepler's law to write

$$
\tilde{U} \sim \frac{M_{2}}{M_{1}} \omega^{2} r^{2},
$$

and grouped the dimensional factors in the first term. The negative sign in equation (22) implies that the gravity waves transport negative angular momentum density. ${ }^{9}$ Zahn (1975, 1977) equates $-L_{H}$ to the tidal torque, $T_{T}$, acting on the primary star. Our expression is a good approximation to his.

The tidal synchronization time, assuming that the star maintains a state of uniform rotation as it despins, is then of order

$$
\tau_{\text {syn }} \sim \frac{I \Omega}{T_{T}},
$$

where $I=f M_{1} R^{2}$ is the moment of inertia of the primary star. It reduces to the more transparent expression

$$
\tau_{\text {syn }} \sim \frac{\Omega(G \bar{\rho})^{7 / 3}}{\omega^{3} \sigma^{1 / 3}}\left(\frac{M_{1}}{M_{2}}\right)^{2} f .
$$

Of course, our point, elaborated upon in $\S$ III $e$, is that the surface layers are despun first, and therefore that the observed rate of synchronization is more rapid than implied by this equation. In this context, we note that the tidal torque depends upon the tidal frequency, $\sigma$, evaluated at the core-envelope boundary, and thus vanishes only when the interior regions of the star have been despun.

\footnotetext{
${ }^{8}$ A rigorous justification of this procedure requires solving the inhomogeneous differential equation that describes the forcing of gravity waves by the tidal potential. This was first accomplished by Zahn (1975).

${ }^{9}$ Of course, this statement is only true if $\sigma>0$.
}

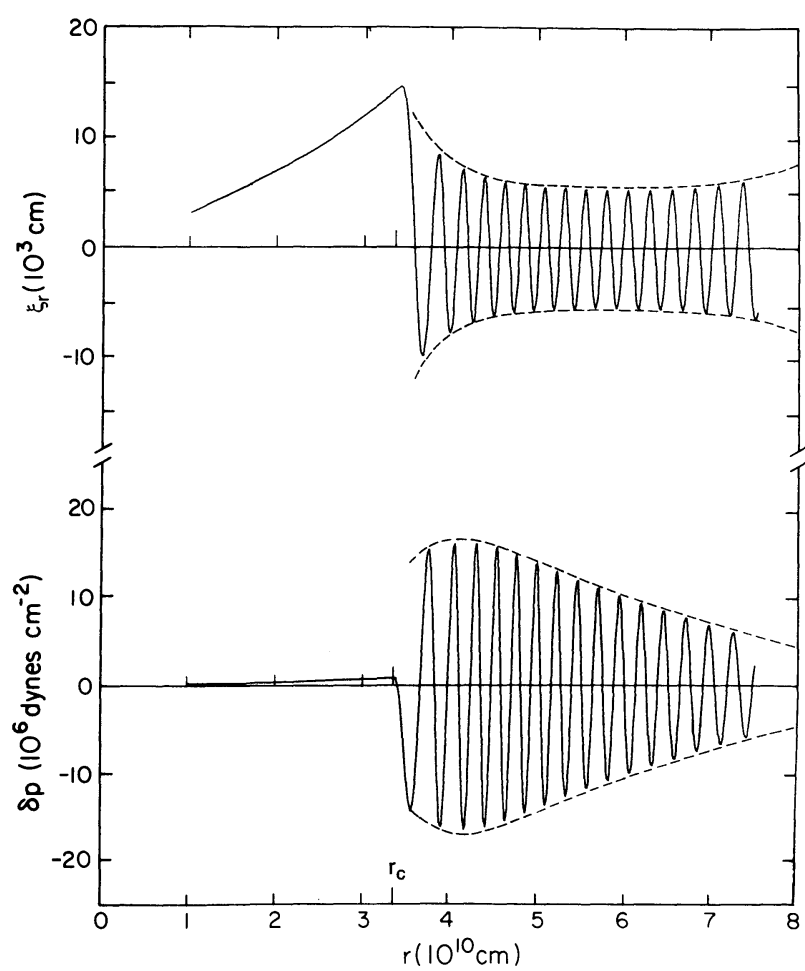

FIG. 1.-Numerical solution of the linearized equations of motion for the dynamical tide in a nonrotating $5 M_{\odot}$ main-sequence star. The core-envelope boundary is denoted by $r_{c}$. The stellar radius is $1.88 \times 10^{11} \mathrm{~cm}$; the tidal frequency $\sigma=2 \times 10^{-5} \mathrm{~s}^{-1}$. Only the homogeneous solution is shown; that is, $U=0$. The radial displacement and pressure perturbations are denoted by $\xi_{r}$ and $\delta p$. The dashed curves indicate the WKBJ amplitudes obtained from equation (21) and are fitted to the numerical solution at large $r$ (after Fig. 7 of Nicholson 1978).

\section{c) Subsurface Reflection of Gravity Waves}

Close to the photosphere $g$ approaches its surface value and $H$ is just a few times larger than the depth, $z$. Gravity waves are evanescent for $H\left|k_{r}\right| \lesssim 1$. Thus, outward propagating gravity waves are reflected where

$$
\frac{z}{R} \sim \frac{\sigma^{2}}{G \bar{\rho}} .
$$

Since the depth of the reflecting layer is proportional to $\sigma^{2}$, radiative damping of the dynamic tide increases in importance with decreasing frequency. Note also that gravity waves of sufficiently low frequency, $\sigma^{2} / G \bar{\rho} \ll H(R) / R$, do not have an outer reflecting layer and propagate into the optically thin layers above the stellar photosphere where they become nonlinear and damp.

\section{d) Comments on Savonije and Papaloizou (1984)}

Savonije and Papaloizou (1984) state that the oscillatory tidal torque density gives rise to a complicated state of differential rotation in the radiative envelope. This contention is in conflict with the theorem we proved in Paper I. Here we expose its flaw.

The total tidal torque may be written as

$$
T_{T}=-\int_{0}^{R} d r r^{2} \int d \Omega \delta \rho(x) \frac{\partial U_{T}(\boldsymbol{x})}{\partial \phi} .
$$


We define the radial tidal torque density to be

$$
\mathscr{T}_{T}(r)=-r^{2} \int d \Omega \delta \rho(\boldsymbol{x}) \frac{\partial U_{T}(\boldsymbol{x})}{\partial \phi} .
$$

The Eulerian density perturbation $\delta \rho=\delta \rho^{\mathrm{eq}}+\delta \rho^{\mathrm{d}}$. Since $\delta \rho^{\mathrm{eq}}$ is orthogonal to $\partial U / \partial \phi$ upon integration over $\phi, \mathscr{T}_{T}(r)$ arises entirely from $\delta \rho^{\mathrm{d}}$. It exhibits an almost harmonic oscillation with phase $\int^{r} d r^{\prime} k_{r}\left(r^{\prime}\right)$ superposed on a slowly varying envelope.

Savonije and Papaloizou's tidal torque density is compatible with ours. However, they err in assuming that this is the only torque density which acts in the star. In fact, the fluid in the primary star is subject to internal stresses associated with its velocity and pressure fields.

The divergence of the $\phi$-component of the Reynold's stress contributes a torque density

$$
\mathscr{T}_{\mathrm{Re}}=-r \frac{\partial}{\partial r} \int d \Omega \sin \theta\left(r^{2} \rho v_{r} v_{\phi}\right) .
$$

The dominant contribution to $\mathscr{T}_{\mathrm{Re}}$ is obtained by inserting $v_{r}^{\text {eq }}$ and $v_{\phi}^{\text {d }}$ into equation (29), and applying the partial derivative with respect to $r$ to the latter. Using equations (9), (11), and (12), and with a small amount of massaging, this term may be case in the form

$$
\mathscr{T}_{\mathrm{Re}} \sim r^{2} \int d \Omega \delta \rho(\boldsymbol{x}) \frac{\partial U_{T}(x)}{\partial \phi},
$$

which is just the negative of $\mathscr{T}_{T}$. Thus, to leading order the torque density associated with the Reynold's stress cancels that due to the external potential.

The torque density due to the perturbed pressure vanishes because

$$
\mathscr{T}_{p}=r^{2} \int d \Omega \frac{\partial \delta p}{\partial \phi}=0
$$

\section{e) Spin Synchronization}

We now have a clearer picture of the principal features of the tidal perturbation, at least in regions of the stellar envelope which are dissipation free. The tidal potential raises a largescale equilibrium tide, and also excites a train of gravity waves at the boundary of the convective core. The tidal potential acting on the density perturbation associated with the gravity waves produces a tidal torque density, $\mathscr{T}_{T}$, that is a rapidly oscillating function of $r$. This is cancelled by another torque density, $\mathscr{T}_{\text {Re }}$, due to the divergence of the $\phi$-component of the Reynold's stress, $\rho v_{r} v_{\phi}$; thus, as demanded by the theorem of Paper I, there is no secular variation of the angular momentum density in the fluid. The dominant contribution to $\mathscr{T}_{\mathbf{R e}}$ comes from the mixed term $\rho v_{r}^{\mathrm{eq}} v_{\phi}^{\mathrm{d}}$. Another term, $\rho v_{r}^{\mathrm{d}} v_{\phi}^{\mathrm{d}}$, is responsible for the angular momentum luminosity, $L_{H}$, carried by the gravity waves. This quantity rises from zero to its full value, $-T_{T}$, within a distance of order $\lambda_{1}$ from $r_{c}$. The angular momentum transferred to the star is ultimately deposited in the fluid where the gravity waves damp.

Of course, the surface layers account for but a small fraction of the star's moment of inertia so they are rapidly despun. The precise rate depends upon the fractional absorption of the incident gravity waves and the efficiency at which angular momentum is transported outward by magnetic stresses and instabilities excited by differential rotation. At first sight it might appear that subsynchronous, or even retrograde, rotation of the surface might result. However, the group velocity of gravity waves vanishes at corotation so the waves cannot reach the surface after it has despun to synchronous rotation. As the waves approach the synchronously rotating layers from below their group velocity slows, their wavelengths diminish and their amplitudes increase. The net result is that the waves are absorbed and deposit all of their negative angular momentum below the synchronously rotating layers. The situation is simpler than before the surface reaches synchronous rotation because the waves are completely absorbed and there is no reflected component (see discussion following eq. [16]).

All this leads to a rather bizarre conclusion. The tidal torque is exerted deep inside the star at the boundary between the convective core and radiative envelope. However, the star spins down toward synchronous rotation from the outside in.

\section{DISCUSSION}

A complete picture of the action of tides in early-type stars must await further advances in our understanding of the evolution of differential rotation in stellar interiors. Differential rotation may be limited by several processes which are hard to identify let alone to quantify. Dynamical instabilities undoubtedly occur if the differential rotation is sufficiently large. However, the static stability of the radiative layers suppresses such instabilities unless the inertial forces associated with the differential rotation are comparable to the gravitational acceleration. Weaker instabilities, which rely on radiative diffusion to mitigate static stability, arise for weaker differential rotation. Unfortunately, their nonlinear behavior is not well understood, so it is difficult to predict how efficient they are in transporting angular momentum out from the stellar interior. Finally, the Maxwell stress associated with an internal magnetic field, if present, might dominate the transport of angular momentum in differentially rotating regions.

It is plausible to expect that during tidal despinning a star will pass through a stage where its surface layers rotate more slowly than its interior. ${ }^{10}$ The demonstration by Giuricin, Mardirossian, and Messetti $(1984 a, b, c)$ that the tendency for synchronization, or pseudosynchronization, is really very much stronger than a straightforward application of Zahn's theory would predict supports this view. In some cases, significant despinning of the photosphere is found for stars whose lifetimes are more than two orders of magnitude shorter than the time scale over which the tidal torque would have been expected to enforce synchronous rotation throughout their interiors.

Future progress in understanding tidal despinning of earlytype stars is likely to be slow. However, we can think of two possible qualitative advances which might occur.

The first involves numerical simulation. There are many features of the hydrodynamics of tidal despinning of early-type stars which make it an attractive problem to study numerically. At first, we could learn a lot from two-dimensional studies. However, three-dimensional simulations will ultimately be required to follow the turbulence which may be excited by instability in regions of large differential rotation.

The second hope for a qualitative improvement in understanding comes from stellar seismology, a field which is now in its infancy. At present, we have no way of predicting the levels to which the normal modes of oscillation of early-type stars are excited. Should they prove to be excited to observable levels, determination of their frequency splittings by stellar rotation

${ }^{10}$ This explanation was anticipated by Zahn (1984). 


\section{GOLDREICH AND NICHOLSON}

might be possible. These splittings would reveal the internal $\therefore$ rotation and provide a direct test of the hypothesis advanced in , this paper.

in!

Iㄴ.

Giuricin, G., Mardirossian, F., and Mezzetti, M. 1984a, Astr. Ap., 131, 152 1984b, Astr. Ap., 134, 365.

. 1984c, Astr. Ap., 135, 393.

Goldreich, P., and Nicholson, P. D. 1989, preprint (Paper I)

Nicholson, P. 1978, Ph.D. thesis, Caltech.

Savonije, G. J., and Papaloizou, J.C. B. 1983, M.N.R.A.S., 203, 581.

Zahn, J-P. 1966a, Ann. Ap., 29, 313.

Peter Goldreich: Mail Code 170-25, California Institute of Technology, Pasadena, CA 91125

PhiliP D. Nicholson: Space Science Building, Cornell University, Ithaca, NY 14853

Zahn, J-P. 1966b, 29, 489
This research was supported by NSF grant AST 86-1299. P. G. is grateful to J. N. Bahcall for his hospitality at the Institute for Advanced Study, where this investigation was initiated.

\section{REFERENCES}

$1966 c, 29,565$.

1975, Astr. Ap., 41, 329

1977, Astr. Ap., 57, 383

. 1984, IAU symposium 105, Observational Tests of Stellar Evolution

Theory. ed. A. Maeder and A. Renzini (Dordrecht: Reidel), p. 379. 